
\title{
Synthesis and Magnetic Properties of Hydrophilic and Hydrophobic Hybrid Nanocomposite
}

\author{
Z. Mitroóvá ${ }^{a, *}$, I. Antal ${ }^{a}$, A. JuríkOváa ${ }^{a}$, M. RAJŇÁK $^{a, b}$, \\ Z. DANKOVÁ ${ }^{c}$, M. TimkO ${ }^{a}$ AND P. KOPČAnsKÝ ${ }^{a}$
}

${ }^{a}$ Institute of Experimental Physics, Slovak Academy of Sciences, Watsonova 47, 04001 Košice, Slovakia

${ }^{b}$ Faculty of Electrical Engineering and Informatics, Technical University of Košice, Letná 9, 04200 Košice, Slovakia

${ }^{c}$ Institute of Geotechnics, Slovak Academy of Sciences, Watsonova 45, 04001 Košice, Slovakia

\begin{abstract}
The magnetic properties of hydrophilic hybrid nanocomposite (multi-walled carbon nanotubes modified with magnetite nanoparticles) and hydrophobic hybrid nanocomposite (multi-walled carbon nanotubes modified with magnetite nanoparticles coated with oleic acid) were studied in the temperature range of 2-290 K and magnetic field up to $6 \mathrm{~T}$. Both samples were characterized morphologically, thermally, and magnetically. Magnetite nanoparticles bounded to multi-walled carbon nanotubes were close to spherical and the particle size was distributed from 10 to $25 \mathrm{~nm}$, as observed by transmission electron microscopy. The thermogravimetric analysis of the hydrophobic hybrid nanocomposite pointed out bounding the oleic acid on the nanoparticles. The hydrophobic, as well as the hydrophilic nanoparticles exhibited a typical superparamagnetic behavior at room temperature. The magnetic measurements at $2 \mathrm{~K}$ indicated ferromagnetic behavior. The value of coercivity of hydrophilic or hydrophobic nanocomposites at $2 \mathrm{~K}$ were $0.018 \mathrm{~T}$ and $0.037 \mathrm{~T}$, respectively. It was found that the presence of the bounded oleic acid results in the decrease in the magnetization of saturation, as compared to the hydrophilic nanocomposite.
\end{abstract}

DOI: 10.12693/APhysPolA.137.973

PACS/topics: carbon nanotubes, magnetic properties and materials

\section{Introduction}

Since the discovery of carbon nanotubes (CNTs) in 1991 [1], various applications have been proposed due to their excellent physical properties. Their application in liquid state for various applications like MRI, EMI shielding, hyperthermia, energy conversion, etc., is subjected to an intensive research. Because of their nature, CNTs are inherently insoluble in most organic and aqueous solvents. Thus, surface modification of CNTs in order to improve the compatibility and solubility has been one of the main research points during the last few years. Noncovalent or covalent functionalization of CNTs can enhance their compatibility and solubility [2]. The modification of CNTs with some organic compounds includes covalent binding of nanoparticles onto CNTs, preparing new multifunctional nanocomposite by dispersing in a chitosanstarch polymer [3-5]. Nowadays it is evident that the $\mathrm{CNT}$ is a supermaterial with a huge range of industrial applications $[6,7]$. Furthermore, many studies have focused on depositing metal or oxide metal nanoparticles on the nanotubes surface [8]. In this paper we report on the preparation and characterization of novel hydrophilic hybrid nanocomposite (multi-walled carbon nanotubes MWCNTs modified with magnetite nanoparticles) and hydrophobic hybrid nanocomposite (MWCNTs modified with magnetite nanoparticles coated with oleic acid).

\footnotetext{
*corresponding author; e-mail: mitro@saske.sk
}

\section{Experimental}

\subsection{Synthesis}

Chemical vapor deposited MWCNTs were purchased from Sigma Aldrich Co. (length from $0.5 \mu \mathrm{m}$ to $2 \mu \mathrm{m}$, outer diameter from $20 \mathrm{~nm}$ to $30 \mathrm{~nm}$, wall thickness from $1 \mathrm{~nm}$ to $2 \mathrm{~nm}$ ). Initially MWCNTs were treated with $150 \mathrm{ml}$ of $65 \% \mathrm{HNO}_{3}$ and sonicated at $35 \mathrm{kHz}$ for two hours. The mixture was filtered and washed with deionized water till $\mathrm{pH}$ reached 6 . The slurry was dried at $100^{\circ} \mathrm{C}$ for $20 \mathrm{~h}$. For chemical oxidation on side walls, the sample was taken in $200 \mathrm{ml}$ of $65 \% \mathrm{HNO}_{3}$ followed by sonication and heating at $80^{\circ} \mathrm{C}$ for $70 \mathrm{~h}$. Then, the mixture was allowed to cool down to room temperature and washed with deionized water till its $\mathrm{pH}$ reached 6 and then dried at room temperature. Other reagents $\left(\mathrm{FeCl}_{3}, \mathrm{FeSO}_{4} \cdot 7 \mathrm{H}_{2} \mathrm{O}, \mathrm{NH}_{4} \mathrm{OH}, \mathrm{HNO}_{3}\right.$, oleic acid) were of analytical grade.

\subsubsection{Synthesis of hydrophilic hybrid nanocomposite}

At the begining, $15.9 \mathrm{~g} \mathrm{FeCl}_{3}$ and $14.0 \mathrm{~g} \mathrm{FeSO}_{4} \cdot 7 \mathrm{H}_{2} \mathrm{O}$ were dissolved in $100 \mathrm{ml}$ of deionized water containing $0.046 \mathrm{~g}$ of oxidized MWCNT and heated under rigorous stirring to $90^{\circ} \mathrm{C}$. Then, $50 \mathrm{ml}$ of $25 \% \mathrm{NH}_{4} \mathrm{OH}$ was added dropwise. The precipitate was heated and stirred for 1 $\mathrm{h}$ to complete the reaction. Next, the mixture was allowed to cool down to room temperature under continuous mixing. The precipitate was separated magnetically and washed with $700 \mathrm{ml}$ of deionized water to remove free ions. The slurry was then freeze dried. 


\subsubsection{Synthesis of hydrophobic hybrid nanocomposite}

At the beginning, $15.9 \mathrm{~g} \mathrm{FeCl}_{3}$ and $14.0 \mathrm{~g} \mathrm{FeSO}_{4} \cdot 7 \mathrm{H}_{2} \mathrm{O}$ were dissolved in $100 \mathrm{ml}$ of deionized water containing $0.046 \mathrm{~g}$ of oxidized MWCNT and heated under rigorous stirring to $60^{\circ} \mathrm{C}$. Then, $4 \mathrm{ml}$ of oleic acid as a primary surfactant and $1 \mathrm{ml}$ of acetone as an emulsifier to form oilin-water emulsion was added. During the further heating at $90{ }^{\circ} \mathrm{C}, 50 \mathrm{ml}$ of $25 \% \mathrm{NH}_{4} \mathrm{OH}$ was added dropwise. The precipitate was heated and stirred 1 hour to complete the reaction. Next, it was separated magnetically and washed with $700 \mathrm{ml}$ of hot deionized water to remove free ions. The slurry was then freeze dried.

\subsection{Characterization of the magnetic nanoparticles}

Transmission electron microscopy (LVTEM) images were obtained in order to analyze the morphology and structure and to measure the particle size distribution of the nanoparticles. Attenuated total reflectance-Fourier transform infrared (ATR-FTIR) spectroscopy (model FTLA2000-100 instrument from ABB) was used to investigate the spectra $\left(520-4000 \mathrm{~cm}^{-1}\right)$ of oleic acid, magnetite, and oleic acid coated iron oxide nanoparticles that were previously dried in the air. The thermal stability of the hybrid nanocomposites was evaluated by means of thermogravimetric (TG) analysis using a TGDTA Setaram SETSYS 16 equipment. The experiments were carried out in nitrogen atmosphere at a flow rate of $100 \mathrm{ml} / \mathrm{min}$ under a heating rate of $10^{\circ} \mathrm{C} / \mathrm{min}$ in the temperature range from $20^{\circ} \mathrm{C}$ to $600^{\circ} \mathrm{C}$. Magnetic properties of the investigated samples were measured by a vibrating sample magnetometer (VSM) installed on a cryogen free high field measurement system from Cryogenic Ltd.

\section{Results and discussion}

Figure 1 shows typical TEM images for oxidized MWCNT and hydrophilic hybrid nanocomposite samples. The intertwined capillaries of oxidized MWCNT have the outer diameter of $20-25 \mathrm{~nm}$. The shape of the magnetite nanoparticles is close to spherical, and the mean particles size is about $20 \mathrm{~nm}$. The main part of them is bonded to the carbon nanotubes.
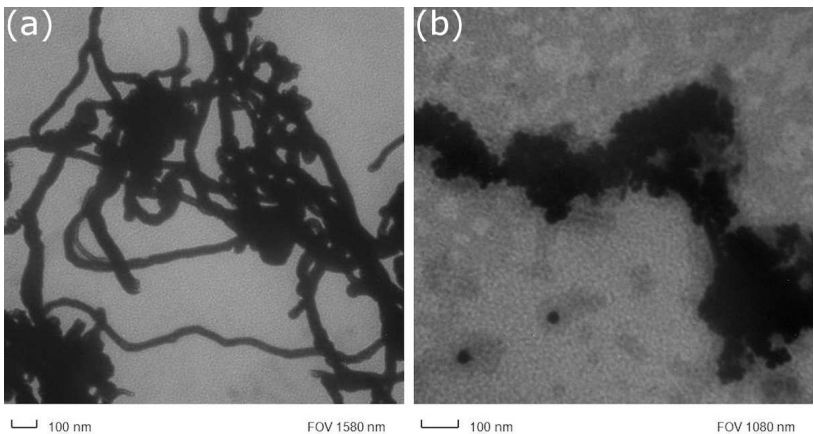

Fig. 1. TEM images of oxidized MWCNT (a), and hydrophilic hybrid nanocomposite samples (b).

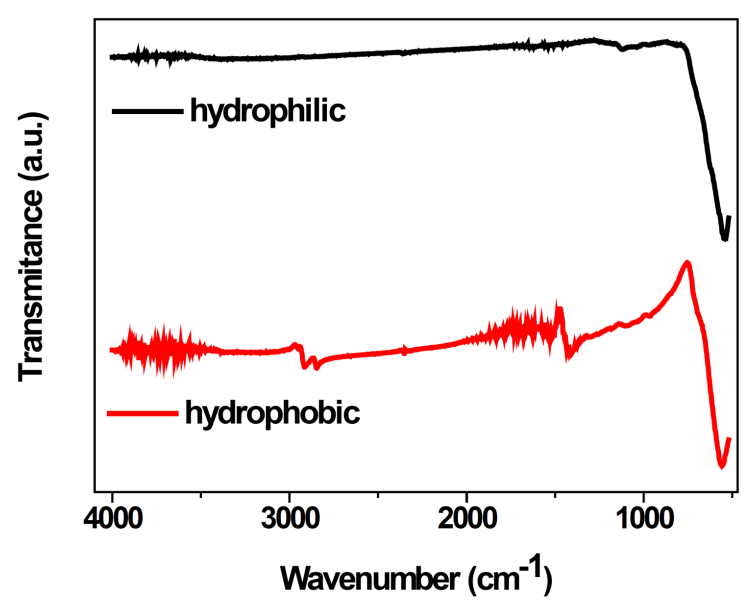

Fig. 2. ATR-FTIR spectra of hydrophilic and hydrophobic hybrid nanocomposite samples.

The typical FTIR spectrum of the pure oleic acid shows the bands at 937, 1284, 1461, $1710 \mathrm{~cm}^{-1}$, and approximately at $2800-2930 \mathrm{~cm}^{-1}$. The peaks at 1461 and $937 \mathrm{~cm}^{-1}$ belong to $\mathrm{O}-\mathrm{H}$ in plane and out of plane bonds. The bands at 1710 and $1284 \mathrm{~cm}^{-1}$ correspond to stretching vibration of $\mathrm{C}=\mathrm{O}$ in oleic acid and the presence of the $\mathrm{C}-\mathrm{O}$ stretch. The bands at 2923 and $2854 \mathrm{~cm}^{-1}$ are attributed to the asymmetric and symmetric stretch of $\mathrm{CH}_{2}$ [9]. Figure 2 includes the ATR-FTIR spectra for synthesized hydrophilic and hydrophobic hybrid nanocomposites. In the spectrum the band at about $550 \mathrm{~cm}^{-1}$ corresponds to the vibration of the $\mathrm{Fe}-\mathrm{O}$ and confirms the presence of an iron oxide. The peak at $1710 \mathrm{~cm}^{-1}(\mathrm{C}=\mathrm{O}$ stretch band of the carboxyl group of OA) was absent in the spectrum but two new bands at 1506 and $1404 \mathrm{~cm}^{-1}$ appeared in this spectrum which are attributed to $\nu_{a s, s}\left(\mathrm{COO}^{-}\right)$stretch vibration band. The asymmetric $\mathrm{CH}_{2}$ stretch and the symmetric $\mathrm{CH}_{2}$ are shifted to 2922 and $2852 \mathrm{~cm}^{-1}$, respectively. These results indicated that oleic acid was coated to the nanocomposites successfully [10].

TG and derivative TG (dTG) curves of the hydrophilic and hydrophobic hybrid nanocomposites, as well as of the pure oleic acid are shown in Fig. 3. No significant weight loss was detected for hydrophilic hybrid nanocomposite over the whole temperature range up to $600^{\circ} \mathrm{C}$ except for the small weight loss of about $4 \%$ up to about $100^{\circ} \mathrm{C}$ due to the evaporation of residual water in the sample. However, for the hydrophobic hybrid nanocomposite the weight loss of about $20 \%$ up to $600{ }^{\circ} \mathrm{C}$ was revealed. Assuming that pure oleic acid is decomposed above the temperature of $300^{\circ} \mathrm{C}$, this weight loss can only be associated with the decomposition of the oleic acid present in the hydrophobic hybrid nanocomposite. Moreover, a shift of the decomposition of the oleic acid in the hydrophobic hybrid nanocomposite to higher temperatures in comparison with the pure one was observed which indicates that the oleic acid is bounded onto the nanoparticles in the prepared hydrophobic hybrid nanocomposite. 

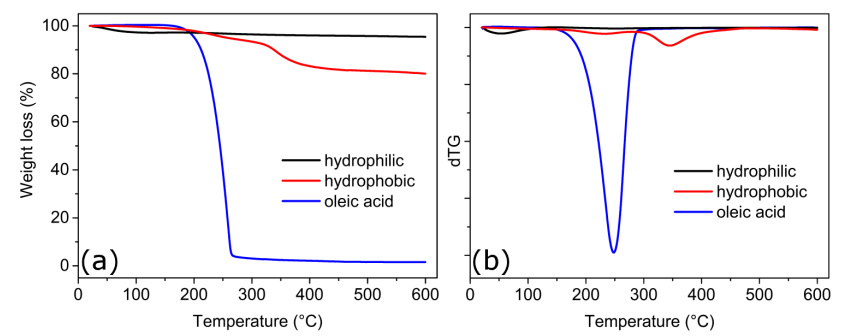

Fig. 3. TG (a) and dTG (b) decomposition curves for hydrophilic and hydrophobic hybrid nanocomposites. The thermograms for the pure oleic acid are added for the comparison.
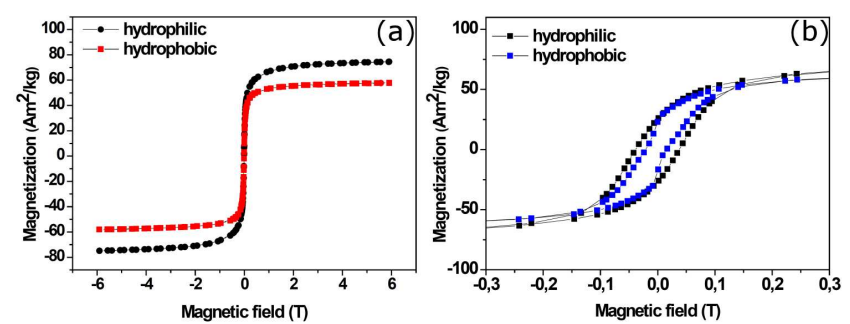

Fig. 4. Magnetization curves of hydrophilic and hydrophobic hybrid nanocomposite measured at $298 \mathrm{~K}$ (a), and $2 \mathrm{~K}(\mathrm{~b})$.

In Fig. 4, one can see the field dependent mass magnetization of the hybrid nanocomposites. The hydrophobic as well as the hydrophilic nanocomposites exhibit a typical superparamagnetic behavior at room temperature. This conclusion follows from the fact that the room temperature magnetization curves show zero hysteresis (Fig. 4a). On the other hand, at low temperature $(2 \mathrm{~K})$ the nanoparticles are in a blocked state when the magnetic moments do not fluctuate any more during the measurement time. This is reflected in the remarkable hysteresis seen in Fig. 4b. The nanoparticle surface modification has a noticeable influence on the magnetic coercivity, as the hydrophobic nanoparticles exhibit $\mu_{0} H=0.036 \mathrm{~T}$, while for hydrophilic nanoparticles $\mu_{0} H=0.03 \mathrm{~T}$. Furthermore, significant effect of surface modification on the magnetization of saturation was observed. While the hydrophilic nanoparticles magnetization of saturation is $84.7 \mathrm{Am}^{2} / \mathrm{kg}$ at $2 \mathrm{~K}$, and $74.7 \mathrm{Am}^{2} / \mathrm{kg}$ at $298 \mathrm{~K}$, the hydrophobic nanoparticles reach decreased values of $67.3 \mathrm{Am}^{2} / \mathrm{kg}$ and $57.7 \mathrm{Am}^{2} / \mathrm{kg}$ at $2 \mathrm{~K}$ and $298 \mathrm{~K}$, respectively. This decrease can be associated with the nanoparticles' surface spin disorder due to the adsorbed oleic acid molecules and due to the diamagnetic contribution from the oleic acid.

\section{Conclusion}

Thermogravimetric analysis as well as infrared spectroscopy of the hybrid nanocomposites confirmed the successful adsorption of oleic acid on the nanoparticles. The amount of oleic acid bounded to the nanoparticles in the prepared hydrophobic hybrid nanocomposite was estimated. From magnetic point of view it was clearly demonstrated that the bounded oleic acid significantly influences the magnetization of saturation of the nanocomposite. Remarkable decrease in magnetization of saturation for the hydrophobic nanocomposite was attributed to the spin disorder effect due to the absorbed oleic acid on the nanoparticle surfaces. In addition, the diamagnetic nature of the oleic acid may lower the total magnetization of the nanocomposite too.

\section{Acknowledgments}

This work was supported by the Scientific Grant Agency of the Slovak Republic Ministry of Education and the Slovak Academy of Sciences under VEGA contract no. 2/0029/19 and by Slovak Research and Development Agency under the Contract no. APVV-18-0160.

\section{References}

[1] S. Iijima, Nature 354, 56 (1991).

[2] C.Y. Hong, Y.Z. You, C.Y. Pan, Polymer 47, 4300 (2006).

[3] T.L. Van, L.N. Cao, T.L. Quoc, T.N. Trinh, N.N. Duc, T.V. Minh, Adv. Nat. Sci. Nanosci. Nanotechnol. 4, 035017 (2013).

[4] X. Ping, C. Daxiang, P. Bifeng, G. Feng, H. Rong, L. Qing, H. Tuo, B. Chenchen, Y. Hao, Appl. Surf. Sci. 254, 5236 (2008).

[5] K Y. Castrejón-Parga, H. Camacho-Montes, C.A. Rodríguez-González, et al., J. Alloys Comp. 615, S505 (2014).

[6] L. Sun, F. Banhart, A.V. Krasheninnikov, J.A. Rodriguez-anzo, M. Terrones, P.M. Ajayan, Science 312,1199 (2006).

[7] D.A. Heller, E.S. Jeng, T.K. Yeung, B.M. Martinez, A.E. Moll, J.B. Gastala, M.S. Strano, Science 311, 508 (2006).

[8] J. He, B. Chen, A.K. Flatt, J.J. Stephenson, C.D. Doyle, J.M. Tour, Nat. Mater. 5, 63 (2006).

[9] P.Ch. Panta, C.P. Bergmann, Nano Res. Appl. 1, 1 (2015).

[10] K. Lai, W. Jiang, J.Z. Tang, RSC Adv. 2, 13007 (2012). 